\title{
QUALITY IMPROVEMENT Improving identification and brief advice for alcohol in hospital patients: a new sustainable model using medicine management technicians
}

\author{
Authors: Emily Walmsley, ${ }^{\mathrm{A}}$ Lindsay Steel, ${ }^{\mathrm{B}}$ Anya Farmbrough, ${ }^{\mathrm{C}}$ Emily Smith, ${ }^{\mathrm{D}}$ Jacqueline Swabe ${ }^{\mathrm{E}}$ and Julia Sinclair ${ }^{\mathrm{F}}$
}

\begin{abstract}
Alcohol identification and brief advice (IBA) is effective for reducing increased risk alcohol consumption, but systematic sustainable screening systems in hospitals are often lacking. University Hospital Southampton NHS Foundation Trust implemented a trust-wide IBA system by integrating it into a patient's medicines reconciliation review undertaken by medicines management technicians (MMTs) on admission. After an initial pilot, this model was rolled out across the trust and has resulted in a substantial increase in the number of patients screened and accurately coded for alcohol use. Surveys of MMTs have shown their confidence and competence in asking patients about their alcohol has increased since implementation.
\end{abstract}

KEYWORDS: alcohol, screening, brief advice, pharmacy technicians, medicines management technicians

DOI: $10.7861 /$ fhj.2020-0203

\section{Introduction}

One in four adults in England drink alcohol at levels which increase their risk of disease placing heavy and increasing burden on hospitals and widening health inequalities. ${ }^{1-5}$ Clinical guidance is for all patients presenting to hospital to be screened for levels of alcohol use. ${ }^{5}$ The NHS long term plan has prioritised the effective management of alcohol-related harms in acute trusts in its prevention programme. ${ }^{6,7}$

Evidence shows identification and brief advice (IBA) is effective in reducing increased-risk levels of drinking. ${ }^{8}$ The Alcohol Use Disorders Identification Test - Consumption (AUDIT-C) is a brief

Authors: A public health registrar, University of Southampton, Southampton, UK; ${ }^{B}$ principal medicines management pharmacy technician, University Hospital Southampton NHS Foundation Trust, Southampton, UK; ' Calcohol clinical nurse specialist, University Hospital Southampton NHS Foundation Trust, Southampton, UK; D pharmacy research fellow, University Hospital Southampton NHS Foundation Trust, Southampton, UK; ${ }^{E}$ lead pharmacist (anti-infectives), University Hospital Southampton NHS Foundation Trust, Southampton, UK; F professor of addiction psychiatry, University of Southampton, Southampton, UK (three questions) validated screening tool for IBA but systematic sustainable screening systems are currently rare in hospital settings. ${ }^{9}$ University Hospital Southampton NHS Foundation Trust (UHS) has a large 1,100 bed NHS hospital which serves a local population of 1.9 million and admits approximately 8,000 patients each month. We sought to develop an innovative sustainable system for delivering alcohol IBA at UHS, which enabled a good, consistent standard over time and integrated it into clinical systems. Here, we describe the development of the model, outline the outcomes over the 3 years of implementation and detail the key challenges and enablers in adopting a model of this nature.

\section{Methodology}

Pharmacy technicians form a small but stable workforce serving all wards across UHS, unlike other clinical staff. They conduct level 1 medicines reconciliation with every patient within 48 hours of admission and were, therefore, identified as being ideally positioned to carry out IBA as part of that process.

In 2015, a 2-week feasibility pilot was undertaken on 56 wards. Medicine management technicians (MMTs) were trained in IBA using the AUDIT-C. Patients identified as drinking at increased risk (AUDIT-C score 5/12 - 9/12) were given brief advice (in the form of an information leaflet). Patients drinking at high risk or dependent levels (AUDIT-C score 10+/12) were referred to the alcohol care team (ACT). The ACT closely supported MMT training and met with them daily to review experiences and concerns. The results were used to develop a business case for an extended 6-month pilot. Based on the average time taken to undertake IBA, the case was made that by employing additional MMTs, all MMTs could undertake IBA as part of their role, leading to a long-term sustainable implementation of the model.

Hospital information systems were adapted to support IBA by enabling recording of AUDIT-C scores electronically. These are visible to clinical teams, incorporated into discharge summaries, and used for accurate coding of alcohol use as a comorbidity by the trust. Referrals to the ACT for patients scored as high risk or dependent are automatically triggered.

Since implementation across the trust in 2016, the provision of IBA by MMTs has undergone continual quality improvement. A series of staff surveys and engagement sessions with MMTs conducted at various times during implementation identified challenges including confidence in asking AUDIT-C and calculating units and concerns about provision of brief advice. 


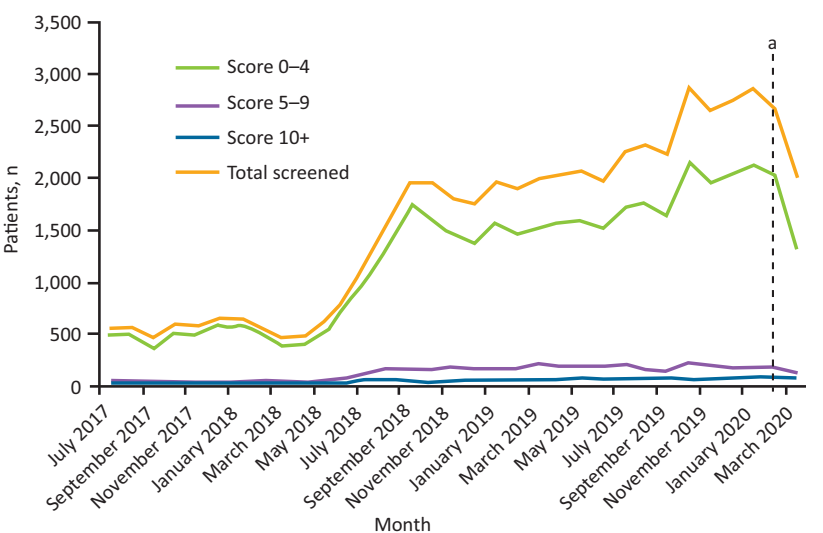

Fig 1. Patients receiving alcohol identification and brief advice at University Hospital Southampton NHS Foundation Trust 2017-2020 and their scores on AUDIT-C. $a=$ changes in screening processes due to COVID-19 procedures.

These have been addressed with tailored training, support from the ACT and resources to support IBA.

\section{Outcome}

Two-week feasibility pilot

Over the 2-week pilot in 2015, 36 MMTs undertook IBA on 602/789 patients they reviewed, adding on average 6 minutes time spent with each patient. Approximately one-quarter of patients were identified as drinking alcohol at increasing or high-risk levels and provided with the appropriate intervention.

\section{Implementation of screening by the MMT}

In-house audit data suggest that fewer than $10 \%$ of medical inpatients were screened for alcohol prior to implementation of the MMT model. Data on screening collected since inclusion of IBA on the hospital information systems demonstrates a significant and sustained increase following implementation of the MMT model and roll out to other areas of the hospital in July 2018 (Fig 1).

The majority (just over $85 \%$ ) of patients screened had an AUDIT-C score of $0-4$ which did not require further action.

\section{Patient and staff surveys}

Short structured interviews with patients on medical wards identified a generally positive view of IBA by healthcare professional such as MMTs, with opinions that hospital is an appropriate place to ask about and address drinking that impacts on health.

Comparison of surveys of MMTs conducted between 2017-2019 as part of implementation suggests that MMTs' self-perceived competence and confidence in asking patients about their alcohol intake and calculating units of alcohol increased. However, there were mixed feelings with some MMTs considering it too personal or intrusive, while others agreed that they were well placed to conduct IBA (Box 1). By the 2019 survey, almost all responses were positive about their alcohol screening role, with a single MMT expressing a wholly negative view about the inclusion of lifestyle advice in their work.
Box 1. Examples of medicines management

technicians' responses to open questions about

their views and experiences of alcohol identification

\section{and brief advice}

'I worry that patients will feel I am asking something too personal or maybe I feel that asking about someone's drinking habits is quite personal. I don't want to make patients feel judged about their lifestyle.' MMT 16, 2017 survey.

'I believe it is an important aspect of patient care as it can highlight potential risks to patient health and offer opportunities for patients to come to terms of their excessive alcohol consumption and then be able to make decisions to lower their consumption.' MMT 14, 2019 survey.

'My confidence is growing in being able to work out the units and I think the more I continue to do so, the more competent I will feel in carrying out this role.' MMT 8, 2019 survey.

'I find the patients that score 5-7 easy to offer advice to and suggesting to these patients they reduce their intake slightly. I find patients that [score] 8-10 are often defensive and say they don't have a problem - and often refuse a leaflet.' MMT 2, 2019 survey.

Confidence in conducting IBA increased with practice and, in most cases, was undertaken comfortably with the support of leaflets, although MMTs felt less confident in some situations (eg patients whose drinking is not easily categorised by the AUDIT-C and offering advice to more complex patients such as those who are bereaved or younger binge drinkers). When asked what worked well, MMTs identified creating a conversation with the patient and explaining why they were being asked about alcohol, the use of information leaflets and unit calculator tools, and having an easyto-use IT system.

\section{Conclusion and next steps}

This case study presents a model for alcohol IBA in an acute trust which is cost-effective, acceptable to patients and sustainable. It has led to a substantial increase in the number of patients receiving IBA trust-wide and accurate coding of alcohol use. This enabled the trust to claim the healthcare resource group (HRG) code tariff better reflecting the complexity of patients with alcohol use disorder.

Despite initial concerns from MMTs about the impact on their workload and it being considered intrusive by some, IBA is now generally accepted and a gradual increase in confidence in delivery has been observed. The success of this model has been attributed to several factors including strong leadership within the MMT team, a multidisciplinary approach including frequent training and ongoing support from the ACT, and an effective IT system.

The process of implementation has not been without challenges. Some patients objected to finding a record of 'harmful drinking' on the discharge summary, so patients are now informed of this in advance (the terms 'increasing risk' and 'higher risk' drinking may be more acceptable). There have also been challenges with other healthcare staff completing IBA as it is not possible to quality assure the screening and advice given. This has been addressed by a hospital-wide message to staff that IBA is now within the job role of MMTs. 
This model was developed initially to address shortcomings in the identification and management of alcohol harms, however, it has also proven invaluable in the delivery of CQUIN 9 (Preventing ill health by risky behaviours - alcohol and tobacco) introduced in 2017, with MMTs systematically conducting the mandated IBA and the IT infrastructure allowing timely monitoring of performance. Next steps include plans to integrate smoking identification and advice treatment into the MMT role in a similar way.

\section{Key points}

$>$ Key enablers included the commitment for IBA in the trust alcohol strategy and the identification of a reliable and stable workforce where additional capacity could be added to include IBA into their role and daily workload.

> Conducting an initial pilot was beneficial in demonstrating feasibility and provide the foundations for a business case, build confidence in the process, and highlight challenges that needed to be addressed before wide-scale implementation.

> Adopting a multidisciplinary approach with close working relationships between MMT and ACT who supported screening with education, training and feedback was invaluable for implementation.

> Integration with the IT systems allows simple and accurate recording and reporting of IBA, automatic referral to ACT when appropriate, and visibility of which patients have and have not been screened.

\section{Acknowledgements}

Part of this work was undertaken as part of a Health Education England (Wessex) funded Quality Improvement Fellowship in 2018/19. Thanks to the MMT team for their involvement.

\section{References}

1 Public Health England. Developing pathways for referring patients from secondary care to specialist alcohol treatment. London: PHE, 2018.

2 World Health Organization. Global status report on alcohol and health 2018. Geneva: WHO, 2018. https://apps.who.int/iris/ handle/10665/274603

3 Phillips T, Coulton S, Drummond C. Burden of alcohol disorders on emergency department attendances and hospital admissions in England. Alcohol Alcohol 2019;54:516-24.

4 Bellis MA, Hughes K, Nicholls ] et al. The alcohol harm paradox: using a national survey to explore how alcohol may disproportionately impact health in deprived individuals. BMC Public Health 2016;16:111.

5 National Institute for Health and Care Excellence. Alcohol-use disorders: prevention: Public health guideline [PH24]. London: NICE, 2010.

6 NHS England. The NHS long term plan. NHS, 2019. www.longtermplan. nhs.uk

7 Phillips T, Porter A, Sinclair ]. Clinical competencies for the care of hospitalized patients with alcohol use disorders. Alcohol Alcohol 2020;55:395-400.

8 Beyer F, Campbell F, Bertholet N et al. The Cochrane 2018 review on brief interventions in primary care for hazardous and harmful alcohol consumption: a distillation for clinicians and policy makers. Alcohol Alcohol 2019;54:417-27.

9 Bohn MJ, Babor TF, Kranzler HR. The Alcohol Use Disorders Identification Test (AUDIT): Validation of a screening instrument for use in medical settings. J Stud Alcohol 1995;56:423-32.

Address for correspondence: Prof Julia Sinclair, Faculty of Medicine, University of Southampton, South Academic Block, University Hospital Southampton, Tremona Road,

Southampton S016 6YD, UK.

Email: Julia.Sinclair@soton.ac.uk

Twitter: @drjmas 